\title{
Análisis de la mejora del aprendizaje tras la aplicación de Kahoot! en una práctica de laboratorio del Grado de Ingeniería Química
}

\author{
Aina Noverques y María Sancho \\ Departamento de Ingeniería Química y Nucelar, Universitat Politècnica de València. Email: ainome@iqn.upv.es
}

\begin{abstract}
The aim of this study is to improve the students' learning in one of the laboratory practices of the subject Chemical Engineering Experimentation II in the Degree of Chemical Engineering of the Universitat Politècnica València. In particular, the study is focused on the practice of Filtration, which studies the characteristics and operating conditions of the process, and its influence on the filtered liquid. During the teaching of this practice, different difficulties were detected in the students when applying and integrating practical learning into the written report that they must deliver as a result. For this reason, this 2019-20 course it has been decided to make use of the Kahoot! tool to, through a test carried out in the practical sessions, try to improve their learning, measured trough the report mark. This paper describes the use of the Kahoot! application and presents and analyzes the results of the report marks to identify points of improvement in the teaching of the practice. In addition, a statistical analysis is shown that confirms the positive influence of the applied innovation.
\end{abstract}

Keywords: learning, Kahoot!, laboratory practice, experimentation, filtration

\begin{abstract}
Resumen
En este estudio se pretende mejorar el aprendizaje de los estudiantes en una de las prácticas de laboratorio de la asignatura de Experimentación en Ingenieria Química II del Grado en Ingeniería Química de la Universitat Politècnica de València. Concretamente, se trata de la práctica de Filtración, en la que se estudian las características y condiciones de operación del proceso, y su influencia en el líquido filtrado. En la docencia de esta práctica se venían detectando diferentes dificultades en los alumnos a la hora de aplicar e integrar el aprendizaje práctico en la memoria escrita que deben presentar como resultado. Por ello, este curso 2019-20 se ha decidio hacer uso de la herramienta Kahoot! para, a través de un test realizado en las sesiones prácticas, intentar mejorar su aprendizaje, medido a partir de las calificaciones de las memorias. En este trabajo se describe el uso de la aplicación y se presentan y analizan los resultados de las calificaciones de las memorias para identificar puntos de mejora en la docencia de la práctica. Además, se muestra un análisis estadístico que confirma la influencia positiva de la innovación aplicada.
\end{abstract}

Palabras clave: aprendizaje, Kahoot!, práctica laboratorio, experimentación, filtración 


\section{Introducción}

En este apartado se describe la situación inicial de la que parte este estudio, el contexto de la asignatura y la motivación de la innovación realizada. Además se muestran los resultados de una revisión bibliográfica sobre el uso de teléfonos móviles y nuevas tecnologías en el aula, particularizando para su aplicación en ingeniería.

\subsection{Descripción de la situación inicial}

\subsubsection{Contexto de la asignatura}

El Grado en Ingeniería Química supone un total de 240 créditos ECTS, y puede realizarse tanto en la Escuela Politécnica Superior de Alcoi (EPSA) como en la Escuela Técnica Superior de Ingenieros Industriales (ETSII), objeto de estudio en esta investigación. Este grado se encuentra acreditado internacionalmente con el sello EUR-ACE lo que supone la certificación de acuerdo a los principios de calidad, relevancia, transparencia, reconocimiento y movilidad del Espacio Europeo de Educación Superior (ENECA.es, 2020).

En el tercer curso, tercer bloque, y de carácter obligatorio en la formación del alumnado, se encuentra la asignatura de Experimentación en Ingeniería Química II (EIQ-II), con un total de 4,5 créditos. Se trata de una asignatura semestral, $100 \%$ experimental, que pretende reforzar los conocimientos adquiridos en diferentes materias como Cinética Química y Catálisis, Operaciones de Separación, Reactores Químicos y Transferencia de Materia.

Experimentación en Ingeniería Química II la componen 8 prácticas diferentes, cada una con un profesorado distinto, experto en cada disciplina. En cada práctica se incluye una sesión de Práctica Laboratorio (PL) y otra Práctica Informática (PI), para el tratamiento de los datos obtenidos experimentalmente. Los alumnos se dividen en grupos de trabajo, que se mantienen a lo largo de toda la asignatura. Al finalizar cada práctica (incluyendo las dos sesiones comentadas), cada grupo de alumnos debe entregar una memoria escrita, con un peso del $6 \%$, sobre la nota global, en la que el total de memorias suponen un 48\%. Para elaborar la memoria, los estudiantes deben seguir el guion proporcionado al inicio de la asignatura, en el que se les indica que debe incluir tanto la propia presentación de datos experimentales y su tratamiento, como una breve introducción teórica, el procedimiento experimental, la gestión de residuos, la información relevante de la ficha de seguridad y conclusiones.

Lo grupos de trabajo en el laboratorio se realizan en función del alumnado de cada sesión, pero no deben superar en ningún caso los 5 miembros por equipo. De esta forma se facilita el trabajo cooperativo, la planificación y gestión del tiempo, y el desarrollo de las competencias básicas de las prácticas en el laboratorio.

Todas las prácticas son entregadas por el alumnado en un máximo de 7 días después de la finalización de la sesión de informática, para su corrección y evaluación por parte del profesorado. Estas correcciones les servirán para el estudio y mejor compresión de los errores cometidos con vistas al examen final de la asignatura, basada en estas prácticas de laboratorio realizadas.

En esta investigación en particular, de entre estas 8 prácticas que componen la asignatura, el caso de estudio se centra en la práctica de Filtración, impartida la primera quincena del mes de diciembre según el calendario de la asignatura. 


\subsubsection{Motivación de la innovación}

La práctica de Filtración objeto de este estudio es la última impartida en la asignatura, por lo que, cuando la realiza, el alumnado ya dispone de al menos otras 6 prácticas corregidas y evaluadas por el resto del profesorado. Así, el nivel de las memorias en cuanto a su formato y presentación suele ser muy satisfactorio. Sin embargo, se viene observando que algunos conceptos teóricos y/o prácticos (como cambios de unidades) presentan dificultades para los estudiantes ya que no los asimilan ni aplican correctamente.

Pese a entregarles la memoria corregida para su estudio antes del examen, algunos de estos mismos fallos se repitieron. Generalmente, y aunque estaba explicado en las correcciones, el alumnado había memorizado todas las partes sin llegar a su comprensión profunda.

Es por esto que se consideró hacer algún cambio para el presente curso 2019-20 que mejorara el aprendizaje de los estudiantes en la práctica de Filtración, concretamente en la aplicación e integración de conocimientos. Para ello, se decidió utiliza la herramienta Kahoot!, una aplicación de móvil que permite hacer test en el aula. Con ella se pretenden reforzar algunos de los conceptos teóricos y prácticos que presentan más dificultades, haciédolo de forma dinámica y mucho más divertida.

\subsection{Uso de móviles en el aula}

En los últimos años se ha producido un gran avance y desarrollo tecnológico en todos los ámbitos de nuestra vida, tanto para el uso cuotidiano como a nivel laboral y profesional. En este caso, la tecnología de la información y comunicación (TIC) supone un cambio profundo en el proceso de enseñanzaaprendizaje, modificando así los roles tanto del profesorado como del alumnado (Moya, 2016). A través del uso de las TIC se fomenta e intesifica la participación del alumnado, se aumenta su capacidad comunicativa y se mejora la relación interpersonal entre ellas y ellos. Diversos estudios destacan un aumento de la motivación e interés por parte del alumnado cuando se aplican las nuevas tecnologías en el aula frente a los sistemas tradicionales de enseñanza de clases magistrales. Las alumnas y los alumnos pasan de ser considerados meros espectadores, sujetos pasivos, que toman anotaciones sin interactuar con el profesorado, a cooperar y participar durante el proceso de aprendizaje (Martínez, 2017).

Las diferentes aplicaciones y sistemas TIC han ido mejorando y simplificando su uso con el paso del tiempo hasta esta última generación de programas y aplicaciones de uso libre como: Kahoot!, Socrative, Quizizz, Etwinning, etc. Todos ellos presentan una interfaz sencilla, que permite interactuar con el alumnado al mismo tiempo que evaluarlo de una forma más práctica, rápida y concreta.

Una de las aplicaciones, Kahoot, objeto de estudio en esta investigación, es considerado una herramienta evaluativa mediante la cual el alumnado interviene de forma plenamente activa en el aula (Fernádez, 2016) permitiendo así una mejor asimilación conceptual de lo aprendido. Se trata de un juego lúdico en el cual se van proyectando las diferentes preguntas propuestas por el profesorado sobre la pantalla del aula. El alumnado, bien mediante el sistema online o bien mediante aplicación en los teléfonos móviles, accede al modelo de cuestionario. Kahoot puede utilizarse de forma individual o en pequeños grupos o parejas, en función del objetivo planteado en cada asignatura por el profesorado. Después de cada una de las preguntas, aparece la puntuación global de todas y todos, dando lugar a un pequeño debate y comentarios sobre la respuesta correcta y las erróneas. Así, se pueden reforzar los conceptos aprendidos y repasar los errores más comunes.

Sin embargo, cabe destacar como inconveniente principal, cierta dificultad añadida a las nuevas tecnologías: el propio acceso a ella, bien sea por la falta de medios materiales (ordenadores, teléfonos 
móviles) así como problemas de conexión de red (García 2017). Este aspecto debe tenerse en cuenta a la hora de planificar cuándo y dónde se empleará el Kahoot!.

En el ámbito de la ingeniería, son numerosos los casos en los que se ha aplicado la herramienta Kahoot!. En asignaturas como "Ingeniería de la Fabricación" (Sellés, 2016) se ha observado un aumento de la participación del alumnado en la clase que, a su vez, conlleva una mejor asimilación de los contenidos y por tanto mejores resultados académicos. En este caso se planteaban sesiones cortas, de menos de 15 minutos con unas 20 preguntas aproximadamente.

Asimismo, esta aplicación puede utilizarse para sondeos sobre los conocimientos previos que tiene el alumnado, tal y como se ha llevado acabo en la asignatura "Ganadería", del Grado de Ingeniería Agronómica (Bartolomé, 2018) de la Universidad de Sevilla. Kahoot! permite realizar preguntas generales, sobre aspectos descriptivos, y otra parte con preguntas teóricas sobre el temario.

Así, el profesorado dispone de una herramienta que les permite conocer a su alumando y adpatar el nivel de enseñanza-aprendizaje en caso de ser necesario.

Esta herramienta también se ha utilizado en la implantación y la mejora de la docencia en inglés en asignaturas como "Ciencia e Ingeniería de los Materiales" e "Ingeniería y Teconología de los Materiales" en la Universidad de Cádiz (Amaya, 2018), en las asignaturas de "Estadística" de diferentes grados de Ingeniería en la Universitat Politècnica de València (Alcover, 2018) así como en el Grado de Adminsitración y Dirección de Empresas, para reforzar los conceptos matemáticos (Jiménez, 2016).

Se observa así la variedad de usos y aplicaciones de la herramienta Kahoot en el ámbito académico de los diferentes grados del sistema educativo de enseñanza superior.

\section{Objetivos}

El objetivo principal de este estudio es conseguir una mejora en el aprendizaje de los estudiantes en la práctica de Filtración de la asignatura de Experimentación en Ingeniería Química II, de tercer curso del Grado en Ingeniería Química en la Universitat Politècnica de València, medido a través de las calificaciones de la memoria escrita de la práctica. Asimismo, se pretende aumentar la motivación y el grado de atención del alumnado durante las sesiones prácticas de la asignatura. Para ello se emplea la aplicación Kahoot!, una herramienta que permite repasar conceptos teóricos y prácticos trabajados en las sesiones prácticas.

\section{Desarrollo de la innovación}

\subsection{Secuencia de aplicación de la innovación}

Para el desarrollo de la innovación se ha llevado a cabo la siguiente secuencia de tareas:

1) Diseño del test

2) Integración del test en la aplicación Kahoot!

3) Realización del test de Kahoot! por los estudiantes

4) Análisis de los resultados del test

5) Análisis de las calificaciones de las memorias 


\subsubsection{Diseño del test}

El test diseñado consta de un total de 7 preguntas, de las cuales 6 son de respuesta múltiple, con 4 opciones diferentes siendo únicamente una de ellas la respuesta correcta. Una de las cuestiones sin embargo es de Verdadero/Falso. El tiempo máximo de respuesta es de 20 segundos para cada pregunta.

Las preguntas, presentadas en orden aleatorio a las alumnas y los alumnos, están divididas en 3 bloques

\section{- Conceptos teóricos}

Pregunta 1a: Qué implica el factor de compresibilidad

Pregunta 1b: Componentes del lecho filtrante

Pregunta 1c: Definición de la relación másica

Pregunta 1d: Qué supone una diferencia de presiones respecto al caudal

\section{- Conceptos prácticos de laboratorio}

Pregunta 2a: Condiciones de presión y caudal trabajado en el laboratorio

Pregunta 2b: Tipo de torta supuesta para el ensayo

\section{- Conversión de unidades}

Pregunta 3: Conversión de unidades

\subsubsection{Integración del test en la aplicación Kahoot!}

La interfaz de la aplicación Kahoot! permite de forma muy intuitiva y sencilla la creación de test y juegos de preguntas. A partir de los comandos de creación de un nuevo cuestionario, esta herramienta posibilita añadir videos, imágenes y enlaces de Youtube para cada una de las preguntas. Permite modificar de forma individual, dentro de un mismo test, el tiempo de respuesta que se le otorga a las y los estudiantes, así como la puntuación de cada pregunta. Además, se pueden seleccionar entre diferentes tipos de preguntas como las de respuesta múltiple (entre 2 y 4 respuestas siendo únicamente una de ellas válida) o de verdadeo o falso.

Una vez integrado el test en la aplicación, se puede almacenar por tiempo indefinido. De esta manera, cada vez que se necesite se puede acceder a él sin problemas. Asimismo, se puede modificar un mismo test tantas veces como sea necesario sin necesidad de crear nuevas versiones.

Cuando el test está guardado, se pueden realizar diferentes pruebas para comprobar el formato y comprobar cómo los estudiantes verían exactamente el test, a modo simulación. Para que los estudiantes puedan acceder al test, se generará un código, a introducir por el alumnado, tal y como se detalla a continuación.

\subsubsection{Realización del test de Kahoot por los estudiantes}

Una vez finalizada la sesión de laboratorio y la de informática, se realiza una pequeña evaluación mediante el Kahoot!. Se informa a los alumnos que ésta es únicamente para repaso de los conceptos aprendidos con el objetivo de mejorar su rendimiento en la realización de la memoria de prácticas. Antes de empezar se les hace una breve explicación del funcionamiento del programa, por si algún estudiante no lo ha utilizado de forma previa en otra asignatura.

El test se realiza por parejas (o grupos de 3 en caso de ser impares) siempre del mismo grupo con el que se han realizado las sesiones prácticas. En total, 29 grupos de alumnos han participado en el test.

A los estudiantes se les proporciona un código de acceso, generado automáticamente por Kahoot!, que han de introducir en la aplicación para tener acceso al test. Cada grupo de trabajo debe identificarse con sus nombres, y el nombre del grupo que consideren. Cuando todas los estudiantes están registrados se procede al inicio del test. 


\subsubsection{Análisis de los resultados del test}

Después de cada pregunta, aparece la respuesta correcta en pantalla así como el número de grupos que han respondido correctamente. Independientemente de los aciertos, se explica la respuesta adecuada y se abre un pequeño debate/aclaración en el que los estudiantes participan actividamente.

Cuando finaliza el test, la aplicación muestra un ránking de resultados y ofrece un documento Excel con las respuestas de cada grupo, permitiendo así su posterior análisis.

En el apartado de resultados se detallan los resultados obtenidos por los diferentes grupos de trabajo.

\subsubsection{Análisis de las calificaciones de las memorias}

Para confirmar si la diferencia que se observe en las calificaciones es estadísticamente significativa, se realiza un análisis discriminante, comparando las calificaciones de las memorias de la práctica de Filtración, con las calificaciones de un trabajo teórico-práctico, realizado por los mismos estudiantes en el $2^{\circ}$ cuatrimestre de $2^{\circ}$ curso, es decir, durante el curso inmediatamente anterior. En el análisis se han comparado, por lo tanto, dos grupos con los mismos alumnos, pero en dos cursos diferentes, tal y como se muestra en la Tabla 1.

Tabla 1. Grupos comparados en el análisis discriminante

\begin{tabular}{cccc}
\hline Grupo & $\begin{array}{c}\text { Número de } \\
\text { estudiantes }\end{array}$ & $\begin{array}{c}\text { Asignatura } \\
2^{\circ} \text { curso }\end{array}$ & $\begin{array}{c}\text { Asignatura EIQ-II } \\
\text { 3er curso }\end{array}$ \\
\hline 1 & 66 & Curso 2017-18 & Curso 2018-19 \\
2 & 63 & Curso 2018-19 & Curso 2019-20
\end{tabular}

\section{Resultados}

En este apartado se detalla el análisis de las respuestas obtenidas a través de la herramienta Kahoo!, y se realiza una comparación entre las calificaciones del alumnado durante el curso 2018/2019 y el 2019/2020.

\subsection{Análisis de las respuestas del test}

A continuación se analiza, para cada bloque de preguntas realizadas, los grupos totales que respondieron correctamente y el porcentaje que esto supone respecto al total de los 29 grupos que han participado en el estudio. Los resultados obtenidos se presentan en la Tabla 2.

Tabla 2. Resultados de las cuestiones del test realizado con Kahoot!

\begin{tabular}{llcc}
\hline \multicolumn{2}{c}{ Cuestiones Kahoot! } & $\begin{array}{c}\text { Total de } \\
\text { grupos con } \\
\text { aciertos }\end{array}$ & $\begin{array}{c}\text { Total de } \\
\text { aciertos (\%) }\end{array}$ \\
\hline \multirow{2}{*}{ Conceptos teóricos } & Pregunta 1a & 26 & 89,66 \\
& Pregunta 1b & 14 & 48,28 \\
& Pregunta 1c & 13 & 44,83 \\
Conceptos prácticos de & Pregunta 1d & 25 & 86,21 \\
laboratorio & Pregunta 2a & 9 & 31,03 \\
Conversión de unidades & Pregunta 2b & 18 & 62,07 \\
\hline
\end{tabular}


Tal y como se observa en la tabla anterior, casi un $90 \%$ del alumnado ha sabido responder qué implica un factor de compresibilidad dado y qué implica una diferencia de presiones respecto al funcionamiento del caudal. Sin embargo, menos de la mitad de los grupos han respondido satisfactoriamente sobre aspectos mucho más concretos y teóricos acerca de los componentes del lecho filtrante y la definición de la relación másica.

En el segundo bloque de preguntas, enfocado a las suposiciones realizadas para los ensayos en el laboratorio, únicamente un $31 \%$ ha sabido en qué condiciones de presión y caudal han estado trabajando en clase mientras que un $62 \%$ ha sabido que inicialmente se había supuesto que la torta a trabajar era incompresible.

Por último, casi un $80 \%$ ha sabido realizar la conversión de las unidades de presión de forma satisfactoria.

Estos resultados muestran que, de modo general, y según los grupos de trabajo, han entendido de forma global el funcionamiento del proceso de filtración. Sin embargo, las condiciones de trabajo o los supuestos de trabajo iniciales no han sido asimilados por el alumnado. Esto podría deberse a que en algunos casos, pese a entender el proceso físico, explicado de forma teórica en otras asignaturas, no ha interiorizado el procedimiento experimental que van a realizar en el laboratorio.

Así, se podría plantear para el próximo curso, el uso del Kahoot! antes de la sesión de laboratorio para que entre todas y todos, puedan entender qué van a hacer durante la práctica y en qué condiciones experimentales van a estar trabajando.

Asimismo, las y los estudiantes manifestaron en una encuesta de satisfacción abierta, también voluntaria, que recomendarían el uso de esta aplicación para el refuerzo de conceptos claves, ya que consideraban que era una herramienta útil y divertida para utilizar en el aula. A partir de estos resultados, se plantean nuevos puntos de mejora para la práctica, poniendo así el foco en todo aquello que las alumnas y los alumnos no han podido asimilar.

\subsection{Análisis de las calificaciones de las memorias}

En este apartado se analizan las calificaciones de las memorias de la práctica de Filtración, comparando los resultados obtenidos en el curso anterior 2018/2019 frente a las de este curso 2019/2020, en el que se ha aplicado la herramienta Kahoot!.

Durante el curso 2018/2019 la nota promedio de las memorias fue de 8,32. La máxima nota fue de 9,70; siendo la mínima de 7. En cambio, durante el curso 2019/2019, el promedio de las notas de las prácticas ascendió hasta un 8,52; siendo la nota mayor de 9,80 y la mínima de 7. En el curso 2018-19, la desviación típica entre las notas fues de un 0,77, mientras que en este curso 2019/2020, ha sido de un 0,90.

Como ya se ha descrito previamente en el apartado 3.1, se realizó un análisis discriminante, comparando las calificaciones de las memorias de Filtración con las de un trabajo teórico-práctico, realizado por los estudiantes en el cuatrimestre anterior. Los resultados de dicho análisis proporcionaron un p-valor igual a 0,0406. Al ser un valor inferior a 0,05, se puede afirmar que la mejora observada en las calificaciones sí es significativa y, por lo tanto, puede estar favorecida por la integración del refuerzo de conceptos mediante la aplicación Kahoot!.

Por lo tanto, en curso posteriores se seguirá trabajando con esta aplicación y su integración en la práctica, para mejorar las dificultades todavía pendientes. 


\section{Conclusiones}

En este estudio se ha analizado la posible mejora de las calificaciones de la memoria escrita de la práctica de Filtración en la asignatura de Experimentación en Ingeniería Química II, de tercer curso del Grado en Ingeniería Química en la Universitat Politècnica de València.

La herramienta Kahoot! ha sido aplicada en tres bloques de preguntas: algunas más teóricas sobre definiciones (4), otras sobre el diseño y las características de la práctica (2) y otra sobre la conversión de unidades. A partir de los datos obtenidos se concluye que, pese a haber entendido algunos de los conceptos fundamentales, el alumnado no ha asimilado las condiciones en las que han estado trabajando en el laboratorio. Esto implica que en la planificación futura de la docencia para los siguientes cursos se debe hacer especial énfasis en estos puntos.

Se ha observado que con respecto al año anterior, existe una mejora de 0,2 puntos promedio de estas calificaciones, que se puede afirmar que es estadísticamente significativa, lo que confirma que el uso de la aplicación Kahoot! puede ayudar a mejorar el aprendizaje en los conceptos tratados en la práctica. Esto también está favorecido por el hecho de que la aplicación aumenta la motivación y el grado de atención del alumnado durante las sesiones prácticas de la asignatura, según la encuesta abierta de satisfacción en la que participaron algunos grupos de estudiantes.

\section{Referencias}

ALCOVER ARANDIGA, R., CALDUCH LLOSA, Á., \& VIDAL, S. (2018). Nos divertimos y aprendemos con Kahoot! en las clases de Estadística. In IN-RED 2018. IV Congreso Nacional de Innovación Educativa y Docencia en Red (pp. 165-175). Editorial Universitat Politècnica de València.

AMAYA, J. M. S., CASTRO, M. P. V., ROBLEDO, D. G., \& ROMERO, P. Implantación y mejora de actividades docentes en inglés mediante metodología AICLE en grados de ingeniería.

ESPINOSA, J., MAS, D., DOMENECH, B., HERNANDEZ POVEDA, C., PEREZ RODRIGUEZ, J., y VAZQUEZ FERRI, C. (2019). Implementación del aprendizaje móvil electrónico y de la ludificación en las prácticas de Óptica Oftálmica I.

FUENTES, M., DEL MAR, M., CARRASCO ANDRINO, M. D. M., JIMENEZ PASCUAL, A., RAMON MARTIN, A., SOLER GARCIA, C., y VAELLO, T. (2016). El aprendizaje basado en juegos: experiencias docentes en la aplicación de la plataforma virtual" Kahoot".

GARCIA GARCIA, D. , CARBONELL VERDU, A., MONTAÑES MUÑOZ, N., QUILES, L., y FOMBUENA, V. (2017, July). Incorporación de la aplicación Kahoot! para la evaluación de las prácticas de la asignatura de "Ciencia de Materiales". In In-Red 2017. III Congreso Nacional de innovación educativa y de docencia en red. (pp. 12091217). Editorial Universitat Politècnica de València.

JIMÉNEZ, A. E. M., GÁMEZ, J. M., y GÓMEZ, J. R. C. (2016). Una propuesta para el refuerzo de conceptos matemáticos a través de Kahoot!. Revista del Congrés Internacional de Docència Universitària i Innovació (CIDUI), (3).

MEDINA, E. B., ALDEA, M. J. A., GUERRERO, Y. M., e IBÁÑEZ, A. H. (2018). Análisis de conocimientos previos sobre Ganadería mediante el uso de Kahoot en alumnos del Grado en Ingeniería Agronómica. EDUNOVATIC 2018, 579.

SELlÉS CANTÓ, M. Á., SÁNCHEZ CABALlERO, S., y PÉREZ BERNABEU, E. (2016). Aplicación de la plataforma KAHOOT en asignaturas de Ingeniería de Fabricación. In In-Red 2016. II Congreso nacional de innovación educativa y docencia en red. Editorial Universitat Politècnica de València. 